\title{
The analysis on effect of thrust constant, spring constant, electrical time constant, mechanical time constant to oscillation displacement of slot-less linear oscillatory actuator
}

\begin{abstract}
This paper presents the analysis on effect thrust constant kf, spring constant ks, electrical time constant Te and mechanical time constant Tm to oscillation displacement of slot-less linear oscillatory actuator (LOA) using Permeance Analysis Method (PAM). The results show that the LOA geometrical structure has high impact on the thrust constant kf, spring constant ks, electrical time constant Te, mechanical time constant $\mathrm{Tm}$ and as a result affect the oscillation displacement $x$. Finally, the analysis presents in this paper can be used to determine the best LOA structure based on the desired thrust constant kf, spring constant ks, electrical and mechanical time constant Te and $\mathrm{Tm}$, and oscillation displacement.
\end{abstract}

Keyword: Linear oscillatory actuator; Thrust constant; Electrical time constant; Mechanical time constant; Spring constant; Oscillation displacement 